\title{
Prevalence of burnout syndrome among Work-From-Home IT professionals during the COVID-19 pandemic
}

\author{
A. Kumaresan ${ }^{a}, *$, Prathap Suganthirababu ${ }^{a}$, Vignesh Srinivasan ${ }^{a}$, Y. Vijay Chandhini ${ }^{a}$, \\ P. Divyalaxmi ${ }^{a}$, Jagatheesan Alagesan ${ }^{\mathrm{a}}$, Surya Vishnuram ${ }^{\mathrm{a}}$, K. Ramana ${ }^{\mathrm{a}}$ and Lavanya Prathap ${ }^{\mathrm{b}}$

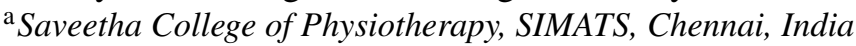 \\ ${ }^{\mathrm{b}}$ Department of Anatomy, Saveetha Dental College, SIMATS, Chennai, India
}

Received 16 July 2021

Accepted 12 October 2021

\begin{abstract}
.
BACKGROUND: Many countries adopted a complete or partial lockdown to prevent the spread of COVID-19, and various professions started to work-from-home. People who work-from-home experience constant stress which leads to burnout syndrome. Burnout can be defined as a chronic stress-induced occupational syndrome resulting in fatigue, decreased job productivity, and poor motivation towards the job.

OBJECTIVE: This research work primarily focuses on exploring the prevalence of burnout syndrome among IT professionals who work-from-home and to determine the impact of burnout in different gender and age-groups during the COVID-19 pandemic.

METHODS: Using snowball sampling method 934 IT professionals were selected based on selection criteria, explained about the study processes, and instructed to submit an online survey consist of Maslach Burnout Inventory scale (MBI) through mail, social media, or through a researcher by direct interview.

RESULTS: Descriptive statistics were used to interpret the data. In 25-30 years age-group, men were affected (93\%) less than women (94\%) and also in 31-35 years age-group, men were affected (96\%) less than women (98\%). Overall burnout scores of men were $94 \%$ and women were $96 \%$. Results indicate female IT professionals were more prone experience burnout syndrome than Male population.

CONCLUSIONS: From the results, it is concluded that $95 \%$ of the IT professionals are suffering from burnout, where women are more prevalent to burnout when compared to male. It is also suggested that an early, routine and frequent assessment of physical status, mental status and burnout syndrome is essential to maintain mental health.
\end{abstract}

\section{Introduction}

The novel coronavirus emerged initially in December 2019 and became a global pandemic disease on April 4th, 2020 affecting many countries. Globally, more than 1 million patients were infected [1], numerous cases faced severe illness and a mortality rate of

*Address for correspondence: A. Kumaresan, Saveetha College of Physiotherapy, Saveetha Institute of Medical and Technical Sciences, Chennai, India. E-mail: kresh49@gmail.com.
$2.3 \%$ were reported [2]. The COVID-19 pandemic has a considerable strain over the economic, social, physical, and mental well-being of the entire population [3].

Considering the mode of disease spread, the World Health Organization recommended a global strategy to control the pandemic through social distancing, sanitizing hands frequently, using face-masks, and isolating the infected person to prevent transmission. Many countries adopted a complete or partial lock- 
down to prevent the spread of disease which resulted in numerous people losing their working environment and supposed to start work-from-home. One of the major professionals who had easy access to work-from-home were Information Technology professionals [4-7].

Though work-from-home was considered to be a better option, significant demerits were hidden and unnoticed. People who work-from-home were in the situation to handle both the job and home responsibilities together, resulting in constant stress leading to work-related burnout syndrome [8]. Burnout can be defined as a chronic stress-induced occupational syndrome that leads to fatigue, decreased job productivity, loss of interest, and poor motivation towards the job. As a consequence, the employee's absenteeism, reduced productivity, and emotional exhaustion might be emerged $[9,10]$.

The professionals who were more prone to burnout were the Information Technology (IT) professionals as they work with increased workload than the normal pattern of working $[11,12]$. Information Technology (IT) is one of the most important sectors of global economy with 10.4 million people worldwide. With the increasing market pressures, long and indeterminate hours of work, lack of time between work and personal life, tight deadlines, and various budgetary constraints leads them to work over-time, and induce chronic stress [13].

Burnout appears abruptly or slowly, with a strong impact on colleagues, friends and even on family. During initial stages of work-from-home, it may be relaxing and stress-free; but this new trend loses fun as the time passes. It might be very difficult to manage the household work and the official work together. Depression, exhaustion, fatigue, anger, and anxiety may begin to appear over this hard work-life as a routine [14-16].

More than half of the working population who work-from-home had reported negative impact on health, with no discrimination of gender and age group [17, 18], but the causal pathways of health issues were not the same. The conservation of resources theory framework states that the salient gender roles or social identities may be threatened in different ways among men and women during their juggling of work and home lives [19].

Burnout and professional fulfillment can be assessed using a questionnaire survey which is specially designed to examine the level of burnout called Maslach Burnout Inventory. Over $90 \%$ of journal articles and literatures used MBI to examine burnout and suggested it as a reliable and valid tool $[20,21]$.

Though most of the previous studies examined the burnout syndrome among IT professionals, burnout due to the emergence of work-from-home during this pandemic situation was not studied yet. Thus, this research work primarily focuses on exploring the prevalence of burnout syndrome among IT professionals who work-from-home and to determine the impact of burnout in different gender and age-groups during the COVID-19 pandemic.

\section{Methods}

\subsection{Participants and procedure}

The snowball sampling method was used to collect the IT professionals who work-from-home and willing to take part in the study from Chennai, India. The study was approved by the Institutional Review Board (05/04/2021/ISRB/FR/SCPT) and Ethical Committee. A total of 1107 participants were invited to participate in this study through E-mails, phone calls, and social media. 934 participants between the age group of 25-35 years were selected based on the selection criteria. The researchers explained the study process in phone calls and instructed to submit an online survey consisting of the Maslach Burnout Inventory scale (MBI) and personal information through mail, social media, or through a researcher by direct interview.

\subsection{Selection criteria}

The IT professionals of both genders between the age group of 25-35 years with normal physical and mental health status were included for the study. Participants with previous mental, physical, or psychological illness; participants under medications or treatment for any chronic illness; participants with chronic medical conditions involving cardiac, pulmonary, orthopedic, neurological, or psychosomatic systems were excluded from the study.

\subsection{Outcome measures}

\subsubsection{Maslach Burnout Inventory scale (MBI)}

The MBI has three sections, namely emotional exhaustion, personal accomplishment, and depersonalization. A participant with a high score in the 1st and 3 rd sections with a low score in the 2 nd section 
Table 1

Percentages and number of participants in each group

\begin{tabular}{lccccc}
\hline & \multicolumn{2}{c}{ Age group } & & \multicolumn{2}{c}{ Gender } \\
\cline { 2 - 3 } \cline { 6 - 6 } & $25-30$ & $31-35$ & & $\mathrm{M}$ & $\mathrm{F}$ \\
\hline No. of & 549 & 386 & & 373 & 561 \\
Percentage & $58.7 \%$ & $41.3 \%$ & & $40 \%$ & $60 \%$ \\
\hline
\end{tabular}

was considered as a person with burnout syndrome [22].

\subsubsection{Personal information}

Age, gender, basic profile, medical history, and personal history including smoking, alcohol, and drugs intake were collected. The participants were grouped based on their gender (male, female) and age groups [25-35].

\section{Data analysis}

Descriptive statistics were used to analyze the burnout syndrome from the selected participants. The mean values and percentages of each gender is calculated based on number of participants in each group. Table 1 represents the percentage calculation and number of participants in each group.

A total score per participant from the three components of MBI was calculated and the burnout was determined based on the total scores. The mean of all scores were also calculated and compared with male and female subject scores.

\section{Results}

\subsection{Sample characteristics}

Among the 1107 IT professionals invited, 934 participants were selected based on the selection criteria and took part in the study. The data collected were used for the descriptive analysis, which revealed that the average age of the sample population was 29.26 years, with more female participants $(561,60 \%)$ compared to male participants $(373,40 \%)$. Around $58.7 \%$ of the participants were aged between 25 to 30 years and $41.3 \%$ were aged between 31 to 35 years. The mean score for all 934 indicates almost $95 \%$ of the IT professionals were affected by burnout syndrome. However, the mean values of each group were studied separately to understand the differences between age-groups and gender (Table 2).
Table 2

Burnout percentage of each group

\begin{tabular}{lccccc}
\hline & \multicolumn{2}{c}{ Male } & & \multicolumn{2}{c}{ Female } \\
\cline { 2 - 3 } \cline { 6 - 6 } & $25-30$ & $31-35$ & & $25-30$ & $31-35$ \\
\hline Emotional exhaustion & $93 \%$ & $96 \%$ & & $95 \%$ & $98 \%$ \\
Personal accomplishment & $91 \%$ & $94 \%$ & & $92 \%$ & $95 \%$ \\
Depersonalization & $92 \%$ & $95 \%$ & & $94 \%$ & $97 \%$ \\
Burnout syndrome & $93 \%$ & $96 \%$ & & $94 \%$ & $96 \%$ \\
\hline
\end{tabular}

\subsection{Burnout syndrome with age groups and gender}

i. The data analysis of 25-30 years age-group showed that the male participants have emotional exhaustion of $93 \%$, personal accomplishment of $91 \%$, and depersonalization of $92 \%$; whereas the female participants have emotional exhaustion of $95 \%$, personal accomplishment of $92 \%$, and depersonalization of $94 \%$ (Graph 1).

ii. The data analysis of 31-35 years age-group showed that the male participants have emotional exhaustion of $96 \%$, personal accomplishment of $94 \%$, and depersonalization of $95 \%$; whereas the female participants have emotional exhaustion of $98 \%$, personal accomplishment of 95\%, and depersonalization of $97 \%$ (Graph 2).

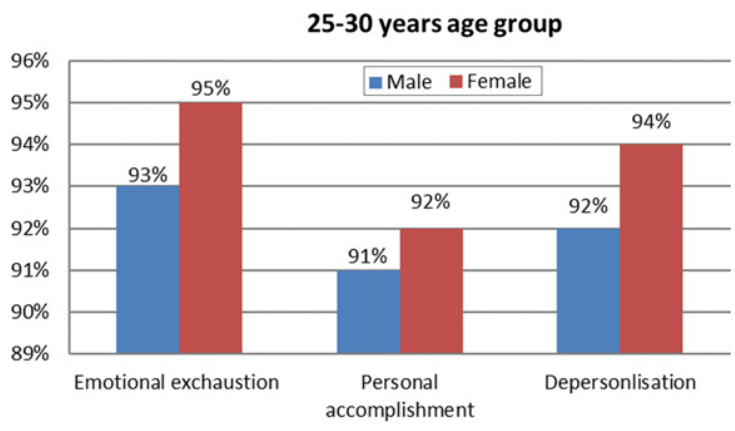

GRAPH- 1. Burnout percentage of male and female participants in the 25-30 years of age category.

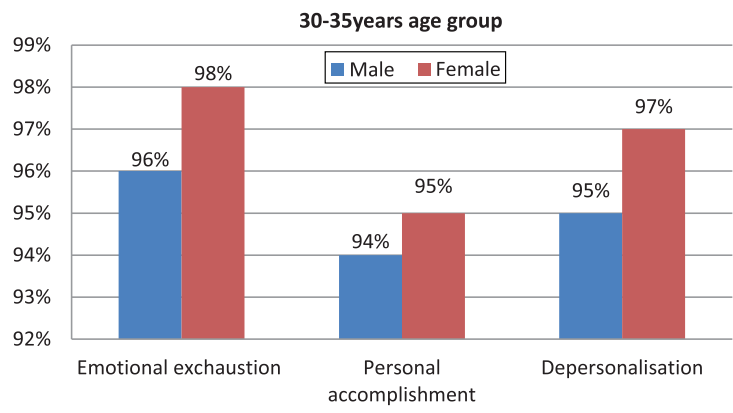

GRAPH- 2. Burnout percentage of male and female participants in the $30-35$ years of age category. 


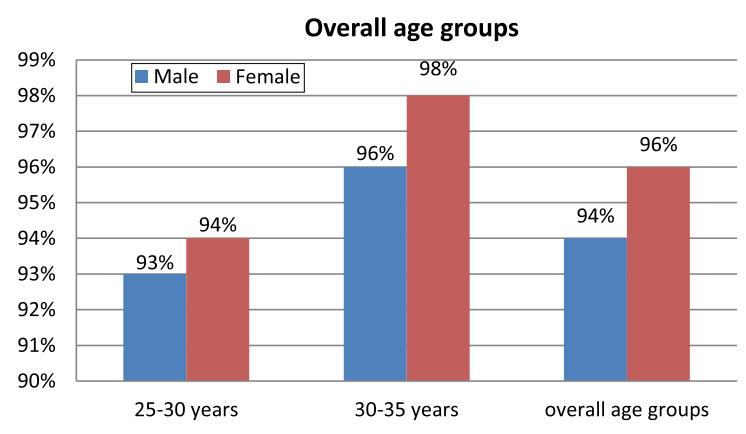

GRAPH- 3. Burnout percentage of male and female participants in the overall age category.

iii. In 25-30 years age-group, males were affected (93\%) less than females (94\%) and also in 31-35 years age-group, males were affected $(96 \%)$ less than females (98\%) (Graph 3). Total participants in general, males were affected by $94 \%$ and female by $96 \%$ measured using MBI scale.

From the above data interpretation, it is understood that the female IT professionals were more prone to have high burnout syndrome than the male population. It is also noted that $95 \%$ of the selected participants (888) were having burnout syndrome with the eldest female group (30-35 years) having more risk to experience burnout syndrome than the other groups.

\section{Discussion}

The current study aimed to explore the prevalence of burnout syndrome in IT professionals who work-from-home during the COVID-19 pandemic. Reviewing the literature suggests that burnout has been documented in various fields and most commonly associated with increased demand to the work [23]. Few studies also supports that IT professionals were vulnerable to work exhaustion [24, 25]. Further, the authors also add that the stressors which excite anger in the working environment could cause burnout syndrome for IT professionals easily.

Sonnentag et al. state that role conflict, job tasks, role ambiguity, and stress have a positive association with burnout [24]. Furthermore, work stressors in the software development profession could be because of the job environment which leads to burnout syndrome. Maudgalya et al. suggest that burnout is not only associated with stress but is also associated with the absence of positive features in the work situation such as complexity of work, high cognitive learn- ing, lack of control, and communication requirements [13]. Huarng et al. concluded that IT professionals have increased levels of burnout, specifically in persons with reduced personal accomplishment, during the systems analysis and maintenance phases [25].

It is evident that IT Professionals experience high stress when compared to other professionals. Though burnout syndrome was prevalent in many other professions it is very much evident in people working in IT $[13,26]$.

Comparing men and women, women were more prevalent to burnout syndrome because the female population has to take care of their household work and to look-after children, especially mothers of children below 5 years were more prone to burnout as they face excessive burden in Indian population. Thus, women spend more time with their children as they graft family work rather than chores [26, 27].

Our findings show that $95 \%$ of the IT professionals who work from home experience high levels of personal and work-related burnout, while most had low rates of client-related burnout and the results of psychological variables showed severe sample levels of anxiety (86.9\%), depression $(88.6 \%)$, and stress (94.4\%). However, $5 \%$ of the experienced professionals with more than 6 years of experience did not seem to have burnout syndrome, and handled their stress level and anxiety with more ease.

The current study enlightens that the burnout syndrome is more prevalent among IT professionals who work from home during the COVID-19 lockdown period whereas women shown to experience higher rates of burnout than men. Considering the prevalence of burnout syndrome in Indian IT professionals, a proper prevention and stress relaxing programs should be framed in order to avoid associated symptoms of the burnout syndrome. Also, this study suggests that an early diagnosis of burnout syndrome among all professions who tend to have more psychological stress in their work environment or workplace than physical stress. Employers and managers should take necessary steps to ensure prevention of burnout among their employees, since this global pandemic has turned many lives uncertain. Employee friendly protocol and time management should be considered and the assigned work must be goal-centric and not time-centric. Goal-centric work schedule can ease up their work load, promotes job satisfaction and reduce overtime work. Further this study is limited to various factors like single urban area, subjective evaluation, and participants age lesser than 35 . Hence, we suggest future trails focusing on 
multicenter studies with large sample size and experimenting treatment protocols will be beneficial.

\section{Conclusion}

From the results, it is concluded that $95 \%$ of the IT professionals suffer from burnout, of which the females are more prevalent compared to males. It is also suggested that an early assessment of burnout syndrome is essential for all professionals to maintain their good physical as well as mental health. Keeping the work environment positive and work-friendly for both genders will provide more ambience, comfort, and a stress-free work zone for the female professionals thereby improving the productivity of their work.

\section{Conflict of interest}

None to report.

\section{Funding}

The authors received no specific funding for this work.

\section{References}

[1] Wu JT, Leung K, Leung GM. Nowcasting and forecasting the potential domestic and international spread of the 2019$\mathrm{nCoV}$ outbreak originating in Wuhan, China: a modelling study. The Lancet. 2020;395(10225):689-97.

[2] Porcheddu R, Serra C, Kelvin D, Kelvin N, Rubino S. Similarity in case fatality rates (CFR) of COVID-19/SARSCOV-2 in Italy and China. The Journal of Infection in Developing Countries. 2020;14(02):125-8.

[3] Godinic D, Obrenovic B, Khudaykulov A. Effects of economic uncertainty on mental health in the COVID-19 pandemic context: social identity disturbance, job uncertainty and psychological well-being model. Int. J. Innov. Econ. Dev. 2020;6(1):61-74.

[4] Gebru AA, Birhanu T, Wendimu E, Ayalew AF, Mulat S, Abasimel HZ, Kazemi A, Tadesse BA, Gebru BA, Deriba BS, Zeleke NS. Global public health significances, healthcare perceptions of communities, treatments, prevention and control methods of COVID-19. Human Antibodies. 2021;29(2):129-37.

[5] Lopez-Leon S, Forero DA, Ruiz-Díaz P. Recommendations for working from home during the COVID-19 pandemic (and beyond). Work. 2020;66(2):371-5.

[6] Omidi L, Moradi G. Risk of COVID-19 infection in workplace settings and the use of personal protective equipment. Work (Reading, Mass.). 2020.
[7] Paraskevopoulos E, Papandreou M. Systematic infection control in Greek Physiotherapy Practices during the COVID-19 pandemic. Work (Reading, Mass.). 2020.

[8] Rodriguez FS, Luck T, Riedel-Heller SG. Enriched environment at work: Disassociated from stress and burnout. Work. 2018;60(1):29-40.

[9] Jeung DY, Kim C, Chang SJ. Emotional labor and burnout: A review of the literature. Yonsei Medical Journal. 2018;59(2):187-93.

[10] Azoulay E, De Waele J, Ferrer R, Staudinger T, Borkowska M, Povoa P, Iliopoulou K, Artigas A, Schaller SJ, Hari MS, Pellegrini M. Symptoms of burnout in intensive care unit specialists facing the COVID-19 outbreak. Annals of Intensive Care. 2020;10(1):1-8.

[11] Vyas L, Butakhieo N. The impact of working from home during COVID-19 on work and life domains: an exploratory study on Hong Kong. Policy Design and Practice. 2020:1-8.

[12] Russo D, Hanel PH, Altnickel S, van Berkel N. Predictors of well-being and productivity among software professionals during the COVID-19 pandemic-a longitudinal study. Empirical Software Engineering. 2021;26(4):1-63.

[13] Maudgalya T, Wallace S, Daraiseh N, Salem S. Workplace stress factors and 'burnout'among information technology professionals: A systematic review. Theoretical Issues in Ergonomics Science. 2006;7(3):285-97.

[14] Sasangohar F, Jones SL, Masud FN, Vahidy FS, Kash BA. Provider burnout and fatigue during the COVID-19 pandemic: lessons learned from a high-volume intensive care unit. Anesthesia and analgesia. 2020 Apr 20.

[15] Rothenberger DA. Physician burnout and well-being: a systematic review and framework for action. Diseases of the Colon \& Rectum. 2017;60(6):567-76.

[16] Crawford ER, LePine JA, Rich BL. Linking job demands and resources to employee engagement and burnout: a theoretical extension and meta-analytic test. Journal of applied psychology. 2010;95(5):834.

[17] Arntz M, Yahmed SB, Berlingieri F. Working from home and Covid-19: The chances and risks for gender gaps. Intereconomics. 2020;55(6):381-6.

[18] Linzer M, Harwood E. Gendered expectations: do they contribute to high burnout among female physicians? Journal of General Internal Medicine. 2018;33(6):963-5.

[19] Langballe EM, Innstrand ST, Aasland OG, Falkum E. The predictive value of individual factors, work-related factors, and work-home interaction on burnout in female and male physicians: a longitudinal study. Stress and Health. 2011;27(1):73-87.

[20] Schaufeli WB, Bakker AB, Hoogduin K, Schaap C, Kladler A. On the clinical validity of the Maslach Burnout Inventory and the Burnout Measure. Psychology \& health. 2001;16(5):565-82.

[21] Coker AO, Omoluabi PF. Validation of maslach burnout inventory. IFE PsychologIA: An International Journal. 2009;17(1):231-42.

[22] Green DE, Walkey FH. A confirmation of the three-factor structure of the Maslach Burnout Inventory. Educational and Psychological Measurement. 1988;48(3):579-85.

[23] Demerouti E, Bakker AB, Nachreiner F, Schaufeli WB. The job demands-resources model of burnout. Journal of Applied psychology. 2001;86(3):499.

[24] Sonnentag S, Brodbeck FC, Heinbokel T, Stolte W. Stressor-burnout relationship in software development teams. Journal of occupational and organizational psychology. 1994;67(4):327-41. 
[25] Huarng AS. Burnout Syndrome among Information System Professionals. IS Management. 2001;18(2):1-6.

[26] Mencher J. Women's work and poverty: Women's contribution to household maintenance in South India. A home divided: Women and income in the Third World. 1988:99119.
[27] Del Boca D, Oggero N, Profeta P, Rossi M. Women's and men's work, housework and childcare, before and during COVID-19. Review of Economics of the Household. 2020;18(4):1001-17. 